\title{
NEURONAL MIGRATION DISORDER - A CLINICAL CASE
}

\author{
Carmen Voicila, Anca Cabat, Raluca Gabriela Ioan \\ "Alfred Rusescu" Institute for Mother and Child Care, Bucharest
}

\begin{abstract}
The authors present the case of a newborn transferred to the Neonatology Section of IOMC Polizu, 43 hours after birth suffering from syndrome of neonatal respiratory distress and suspicion of maternal - fetal infection, extensively investigated during hospitalization for persistency of muscle tone disorder which could not be explained by asphyxia at birth, diagnosed after MRI with right periventricular heterotopia associated with left temporal porencephalic cyst and schizencephaly on the same side.
\end{abstract}

Keywords: cortical heterotopia, MRI, central cause hypotony

\section{INTRODUCTION}

Neuronal migration represents the process by which nervous cells move from their formation place - ventricular and periventricular germinative areas - to the final location in the CNS, where they remain all along their lives. (1) Their placement in different layers of the cortex is genetically determined. (2) There are two types of neuronal migration currently defined: radial and tangential.

Alteration of the normal process of migration (M3 - M5 of pregnancy) and cortical neuronal organization (M5 - neonatal period) leads to brain structural malformations grouped under the denomination of neuronal migration disorders. (1)

By using clinical and neuroimaging criteria, and genetic characteristics these have been grouped into:

- Lissencephaly - agyria/pachygyria complex: absent/partially developed cortex gyri and fissures ("lean cortex" aspect/wide gyri, low in number). From a histologic point of view, it divides into type I (the classic type - Bielschowski) and type II (the Walker - Warburg syndrome). From a clinic point of view, there is encephalopathy with severe axial hypotony. Death occurs during the first months of life.
- Polymicrogyria: high number of small foldings on the cortex. It occurs most frequently within the middle cerebral artery.

- Schizencephaly: discontinuity of the gray matter spread all along a hemisphere, at the level of the central Rolando sulcus or of the Sylvius sulcus.

- Neuronal heterotopias: groups of normal neurons which haven't migrated towards the cortex (periventricular) or stopped from migrating towards the cortex (subcortical). The mechanism is insufficiently known, it is most probably about a disorder in the apoptosis (the mechanism of cell death), genetically programmed. This is considered to be involved in the epilepsy etiopathogeny.

- Cortical disgenesia/focal cortical dysplasia: cortical areas with a disorganized structure. From a clinic point of view, the image is dominated by focal epilepsies with signs of neurological deficiency. (2)

Etiology of these malformations is not fully determined as both genetic (structural molecular defects that condition neuronal migration) and vascular, toxic or infectious factors (e. g. CMV infection in M 3-4 of pregnancy) (1) can be involved. 


\section{CLINICAL CASE}

Name: M.L.G.

Gender: Male

Date of birth: 3/22/2014, 16:30

GA: 38 weeks

BW: 3300 g H: 50 cm, CP: unknown PI 2.64

(harmonious)

\section{CAH}

Mother: 25, IIIG, IP (one spontaneous abortion during the $1^{\text {st }}$ trimester, unidentified cause and one requested abortion), shop attendant, without exposure to toxic environment, positively healthy, smoker (including during the pregnancy duration), blood type AII positive Rh.

Father: positively healthy, blood type AII positive Rh.

Both deny family antecedents of epilepsy or other neurologic diseases, as well as consanguinity.

\section{Pregnancy}

LM: 6/27/2013, LE: first month, FFM: 3 months, treated by the gynecologist doctor, monthly echographies, including the fetal morphology (all within normal limits), TORCH screening done - without severe infections, urinary tract infection with $\mathrm{E}$. Coli during the $2^{\text {nd }}$ trimester treated with Augmentin po, control cultures positively negative, without any other medication during pregnancy.

\section{Birth}

C-section from cranial presentation, AS 8. The baby was intubated a few hours after birth for Syndrome of Respiratory Distress (SRD) with progressive deterioration and received a surfactant as well as treatment with parenteral antibiotic. From a paraclinical point of view: high level of procalcitonine. It was transferred to the IOMC Polizu Clinic after 43 hours of life.

\section{Clinical investigation upon hospitalization:}

Normally weighted newborn, severe general condition, red teguments with mild icterus, generalized edema, FA with normal pressure, rhythmic heart noises on the left of the sternus, VA $150 \mathrm{bpm}$, Tam $30 \mathrm{mmHg}$, symmetrical MV, bilateral rhonchi, Sp O2 96\% (FiO2 40\%), present spontaneous breathes, supple tummy, touchable urinary bladder, low tone and reactivity - sedated.

\section{Anamnesis and clinical diagnosis}

Severe cardiocirculatory insufficiency. SRD severe respiratory insufficiency.

\section{Paraclinical investigations upon hospitalization}

Hemogram: Hb 14g/d, Ht 42.6\%, 18020 le/mmc, $358000 \mathrm{trb} / \mathrm{mmc}$, biochemistry: glycemia $104 \mathrm{mg} /$ dl, ALT $41 \mathrm{U} / \mathrm{L}$, crea $1.3 \mathrm{mg} / \mathrm{dl}$, urrhea $37 \mathrm{mg} / \mathrm{dl}$, Ca $7.2 \mathrm{mg} / \mathrm{dl}, \mathrm{C}$ reactive protein 0.8 , TBi $8.46 \mathrm{mg} /$ dl, DBi $0.22 \mathrm{mg} / \mathrm{dl}$, CI $98 \mathrm{mmol} / \mathrm{L}, \mathrm{K} 4.4 \mathrm{mmol} / \mathrm{L}$, $\mathrm{Na} 132 \mathrm{mmol} / \mathrm{L}$. Peripheric and central cultures negative. TFE: VLS with "snow flake" aspect. Subependymal left cyst.

Biparietal hyperecogenity at the level of the Sylvius sulcus.

\section{Evolution and treatment}

Newborn accepted in the IC, mechanically ventilated, with parenteral antibiotic treatment (Meronem and Vancomicine), inotrope support (Dopamine and Dobutamine), total parenteral nutrition, urinary bladder catheterization, monitoring of the vital functions, TA, diuresis. The evolution was slowly favorable with the cancellation of the ventilating support after 6 days, pev suppression of Dopamine and Dobutamine after 3 days and of sedation with Dormicum iv after 6 days.

Neurologic evolution remains unsatisfying, with hypotony and hyporeactivity while lacking sedating treatment, with difficulties in baby bottle feeding (very low sucking reflex). Control ETF reconfirms the presence of hyperechogenicities at the level of the Sylvius sulcus. Native cerebral MRI is decided and the conclusion is: subcortical heterotopy with a curved aspect, at the level of the internal wall of the posterior conus. Arachnoid cyst on the left anterior temporal pole.

In this context a neurologic checking takes place and shows: "Moderate central hypotonic syndrome. Multiple group modifications: left temporal porencephalic cyst associated with schizencephaly on the same side. Inter and periventricular VLD heterotopy". Periodic neurological monitoring is recommended.

The newborn was released from hospital 22 days after hospitalization with a general good condition, balanced from the cardio-respiratory, digestive, and renal points of view with a slightly diminished reactivity and moderate axial hypotony. Naturally fed with breast milk pumped in the baby bottle (it sucks insufficiently from the breast, getting tired). Worth to be mentioned: no convulsive episode were present during the hospitalization. Auditive screening determined bilateral hypoacusis for which he was directed to the National Institute of Phonoaudiology in Bucharest. It was included in a periodic monitoring program at IOMC Bucharest. 


\section{Discussions}

Disorders occurring during the migration period determine heterotopias. These are stops of nervous cell groups in the white substance in several locations, on their way towards the cortex.

Ventricular heterotopias is a rare illness. Its occurrence is unknown (3). It can be associated with anomalies of chromosome 5, mutations of genes ARFGEF2 (recessive autosomal transmission) or FLNA (X-linked transmission, and in this case boys present much more severe symptoms than girls). In $50 \%$ of the cases the mutation is transmiteed from the infected mother, but new gene mutations can also occur, rarely. (3)

Neuronal migration defects are the grounds of numerous disorders of psychomotor development and it is estimated that approximately $40 \%$ of the babies suffering from treatment proof epilepsy present such a cerebral abnormality. Symptomatology also includes: axial hypotony, mental retardation, growth failure, feeding difficulties, lymphedema, microcephaly, motor dysfunction. These babies usually have a normal phenotype. (4)

The disease is diagnosed when convulsions show up in the clinical condition. In rare cases an MRI examination is practiced for another disease and the diagnosis is thus completed.

Most of these malformations are detectable by an MRI examination. Investigations can be completed with EEG in order to monitor the convulsions and EMG to determine the muscle tone. (5) Cerebral CT has a normal aspect. (2)

Before births, the disease is under diagnosed. It can be skipped during fetal echographies. It is suggestive for the positive echographic diagnosis to take images of the lateral ventricles of a square/ rectangular shape or with irregular margins. (6) Fetar MRI confirms it.
The treatment is in most of the cases symptomatic and supportive - physio-kinetotherapy, occupational therapy, speech therapy, anti-convulsion medication, surgical therapy for treatment proof epilepsies being reserved to selected cases. (4)

The prognosis of patients with syndrome of abnormal neuronal migration is variable and depends on the affected cortical area, on the type of the lesion and the degree of the neurologic deficiency. There can be a minor neurologic disorder or, in other cases, an extremely severe disorder, leading to measures of intensive care to support the vital functions. (4) The syndrome can be associated with other congenital abnormalities/cerebrla malformations, complications which affect the prognosis (7). On a long term, evolution is unfavourable, with a treatment proof epilepsy, wih more and more often crises, till status epilepticus and death.

\section{Case particularity}

Establishing the cerebral malformation diagnosis at the newborn age, MRI confirmation of this diagnosis is usually determined at the age of 1 year when the clinic/neurologic status is finalized.

Lack of risk factors: young parents, no consanguinity, no exposure to environment teratogeneous factors, no infections belonging to the TORCH specter during pregnancy, no epilepsy history in the family or other neurologic disorders.

Complex cerebral malformation - association of periventricular neuronal heterotopy with schizencephaly and porencephalic cyst.

Lack of convulsive manifestations during hospitalization, in the context of coexisting metabolic and electrolytic disorders and circulatory instability.

\section{REFERENCES}

1. Dr. Plesca D., Dr. Teleanu R., Prof. Dr. Dragomir D. Tulburari de migrare neuronala, revista_de_pediatrie.tripod.com

2. Malformatiile sistemului nervos central, 2011, https://ro.scribd.com/ doc/CAP-II-Malformatiile-sistemului-nervos-central.

3. Periventricular Heterotopia - http://ghr.nlm.nih.gov/condition/ periventricular-heterotopia

4. Neuronal Migration Disorders - www.rightdiagnosis.com

5. Great Ormond Street Hospital - www.gosh.nhs.uk/medicalinformation/neuronal migration disorder

6. Periventricular nodular heterotopia on prenatal ultrasound and magnetic resonance imaging - Ultrasound Obstet Gynecol. 2013 Aug; 42(2):149-55. doi: 10.1002/uog.12340.

7. G. d'Orsi. Clinical features and long term outcome of epilepsy in periventricular nodular heterotopia. Simple compared with plus forms, J Neurol Neurosurg Psychiatry 2004;75:873-878 doi:10.1136/ jnnp.2003.024315-http://jnnp.bmj.com/content/75/6/873.full 